\title{
Wearable Haptic Based Pattern Feedback Sleeve System
}

\author{
Anuradha Ranasinghe, Kaspar Althoefer, Prokar Dasgupta, Atulya Nagar, and \\ Thrishantha Nanayakkara \\ Liverpool Hope University, Liverpool, United Kingdom \\ Queen Mary, University of London, London, United Kingdom, \\ MRC Centre for Transplantation, DTIMB \& NIHR BRC, Kings College London, \\ United Kingdom \\ Kings College London, United Kingdom \\ \{dissana@hope.ac.uk, k.althoefer@kcl.ac.uk, prokar.dasgupta@kcl.ac.uk, \\ nagara@hope.ac.uk, thrish.antha@kcl.ac.uk\},
}

\begin{abstract}
This paper presents how humans trained in primitive haptic based patterns using a wearable sleeve, can recognize their scaling and shifting. The wearable sleeve consisted of 7 vibro-actuators to stimulate subjects arm to convey the primitive haptic based patterns. The used primitive haptic patterns are the Gaussian template $(\mathrm{T})$, shifted right (R), shifted left (L), half Gaussian (H), and shrink (S) hereafter denoted by templates. The results of this paper would give an idea as to how humans mentally construct the cutaneous feedback in different scenarios such as shifting and scaling with respect to trained patterns and how they recognize all trained patterns when played randomly. These insights will help to develop more efficient haptic feedback systems using a small number of templates to be learnt to encode complex haptic messages. Therefore, the results provide new insights and design guidelines/algorithm to convey messages encoded in vibro-tactile actuator arrays specially in where vision and audition are less reliable scenarios like search and rescue, factories. For example, the results would be used to convey a message to the human to give an idea of the shape and stiffness of obstacles that come into contact with the robot during haptic based guiding in low visibility conditions in human-robot interactions.
\end{abstract}

Keywords: Haptics, Human-robot interactions, vibro-actuator arrays, guidelines and algorithm

\section{Introduction}

Haptics would be the best way to convey messages in critical tasks to provide spatial information [1]. Some of the studies demonstrated that haptic perceptions can be used to assist humans in navigation in unfamiliar environments [2], [3]. Therefore, it is important to understand how humans perceive haptic feedback patterns using primitive haptic based templates. The results would give us an 
insight as to how to convey messages to humans when they work in noisy or uncertain environments like factory or search and rescue.

There have been some studies demonstrating that haptic feedback can be used to navigate humans [4], [3], [5]. Vibro-actuators have been used for different purposes to convey messages to humans. For example, the study in [5] presented an active belt which is a wearable tactile display that can transmit multiple directional information in combination with a GPS directional sensor and 7 vibro-actuators. Furthermore, in some studies vibro-tactile displays have used to improve the quality of life in different ways such as reading devices for those with visual impairments [6] to provide feedback of body tilt [7], balance control and postural stability [8], and navigation aid in unfamiliar environment [9]. Until now there have been many studies that used vibro-actuator belts for different purposes. However, this paper attempts to understand how to use vibro-tactile actuator arrays to understand representation of distributed haptic feedback which can be used to convey messages to humans.

Amplitude has been widely used to stimulate the human skin in most of the previous studies [10], [11], [12], [13]. However, we argue that frequency would be better for persistent perception. The monotonic nature in amplitude could effect humans' responses.

It has been shown that humans learn movements through flexible combination of primitives that can be modeled using Gaussian like functions [14]. Therefore, in this paper we try to understand whether human somatosensory system also uses primitive patterns that can be modeled using Gaussian like functions to represent haptic perception. These insights will help to develop more efficient haptic feedback systems using a small number of templates to be learnt to encode complex haptic messages.

Basic two scientific questions are tested in this paper. 1) whether the human somatosensory system uses primitive patterns that can be modeled using Gaussian like functions to represent haptic perceptions, 2) whether these primitive representations are localized (cannot be shifted along the skin) and magnitude specific (cannot be scaled).

Therefore, to test those questions, the experiments were carried out to study humans' ability 1) to generalize (scaling/shifting) the trained primitive vibroactuator array templates (T, TR, TL, THA), and 2) to recognize trained these templates and their inverse even they played randomly. Therefore, this is the first paper that attempts to show how the primitive haptic based patterns are represented by humans. The results of this paper would give an idea as to how humans construct the cutaneous feedback in different messages/scenarios. The results would help us to understand what the sensitive geometrical shapes are when we need to code haptic messages to humans in noisy crowded areas such as factory, search and rescue via cutaneous feedback. The possible applications could be human-robot interactions in uncertain environments, noisy situations like factories to convey messages to the humans. 


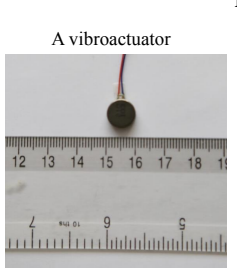

A wearable haptic sleeve with 7 Pico Vibe $10 \mathrm{~mm}$ vibroactuators

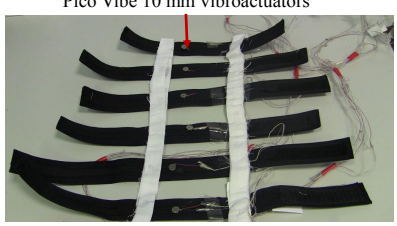

C

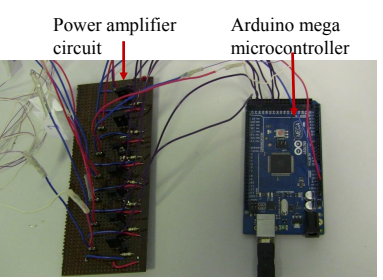

Fig. 1. Hardware design for wearable haptic sleeve: A) Pico Vibe $10 \mathrm{~mm}$ vibro-actuator, B) A wearable vibro-tactile actuator arrays with 7 Pico Vibe $10 \mathrm{~mm}$ vibro-actuator motors, and C) Arduino Mega motherboard and power amplifier circuit to generate different intensity patterns.

\section{Materials and Methods}

The results of three experiments would answer the following scientific questions: Experiment 1: How humans generalize a Gaussian template in scaling and shifting, and Experiment 2: How humans can recognize trained templates even they

A

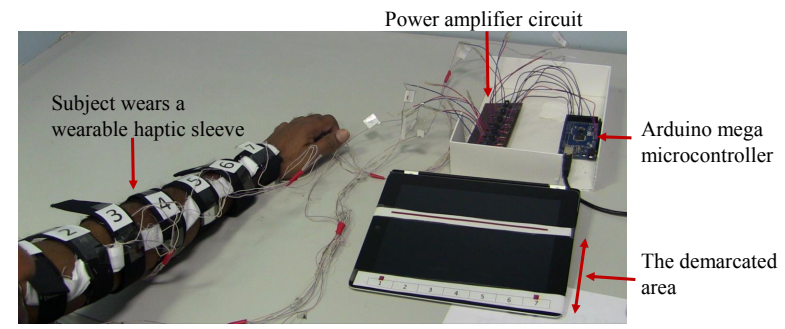

B

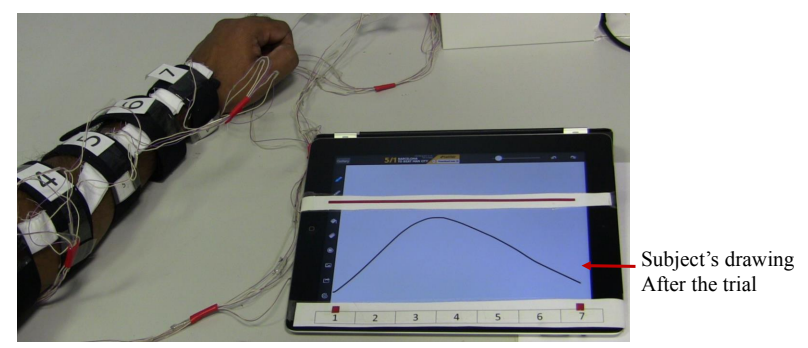

Fig. 2. An experimental trial: A) The subjects wear a wearable haptic sleeve with 7 Pico Vibe $10 \mathrm{~mm}$ vibration motor. The drawing area is demarcated and used hardware is shown on Fig. 2A, and B) The subject drew the intensity felt from the vibro-tactile actuator arrays was during the trial on ipad. Draw free app (Apple Inc) software is used as a drawing tool. 
are presented in a random order given by a set of discrete vibro-actuators on the arm.

Pico Vibe $10 \mathrm{~mm}$ vibration motor - $3 \mathrm{~mm}$ type (Precision Micro-drives) in Fig. 1A was used to make wearable haptic based pattern feedback system as shown in Fig. 1B. There are 7 Pico Vibe $10 \mathrm{~mm}$ vibro-actuators arranged in equal distance $(7 \mathrm{~cm})$ in the array as shown in Fig. 1B. The 7 Pico Vibe 10 $\mathrm{mm}$ vibro-actuators are attached to the seven belts which can be adjusted by strapping securely to arm of the different subjects. The different intensities for the vibrators are generated by Arduino Mega motherboard and the amplitude was modulated by a simple power amplifier circuit as shown in Fig. 1C.

\subsection{Haptic primitive templates generation}

The standard Gaussian function was used to generate templates. The templates were generated by $y=\operatorname{gaussm} f(x,[\operatorname{sig}, c])$ by MATLAB $2014 \mathrm{~b}$, where $s i g=s t d$, and $c$ is the center of the distribution. The sig for pattern T, TR, TL, and THA

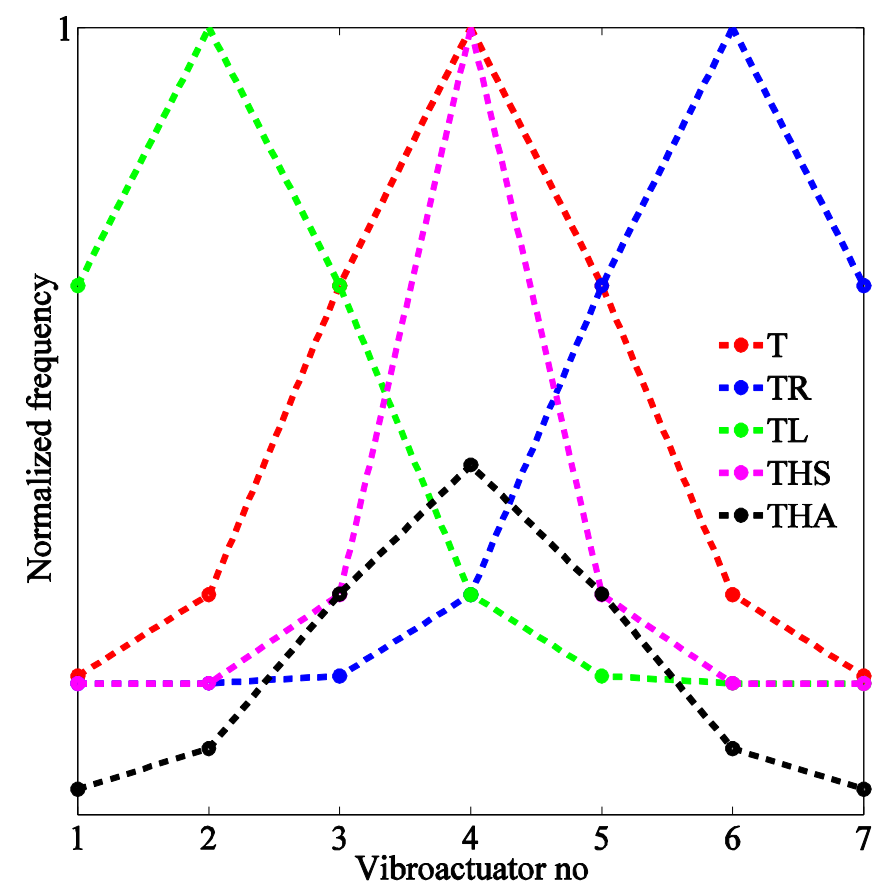

Fig. 3. The templates for experiment 1 and experiment 2: T- Gaussian Template. The patterns was generated by gaussmf (MATLAB R2012b) with the standard deviation 1 for T, TR, TL, and THA. The standard deviation 0.5 was used for THS. 
Table 1. The Intensities $(\mathrm{Hz})$ for different templates in experiment 1 and experiment 2

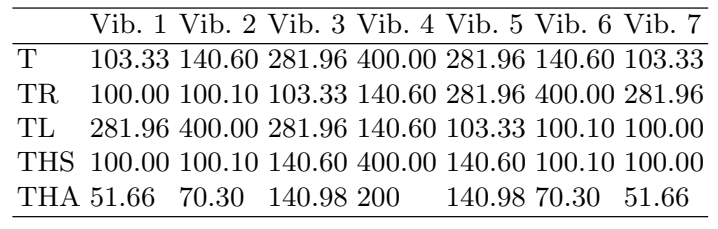

Table 2. The order for different templates in experiment 1 and experiment 2

\begin{tabular}{lll}
\hline & Exp 1: order & Exp 2: order \\
\hline T & $1-5,11-14,20-23,29-32,38-41$ & $1-5,21,29,32,34,38,41$ \\
TR & $6,10,18,27,33,45$ & $6-10,23,27,31,33,39,42$ \\
TL & $7,17,24,35,37,44$ & $16-20,22,26,28,35,37,44$ \\
THS & $9,16,26,28,34,42$ & THS was dropped in Ex2: \\
THA $8,15,19,25,36,43$ & $11-15,24,25,30,36,40,43$ \\
\hline
\end{tabular}

are 1 and 0.5 for THS respectively. Moreover the amplitude of the THA was maintained at half of the rest of the templates.

\subsection{Experimental procedure}

Subjects wore the haptic based pattern feedback sleeve as shown in Fig. 2A. Subjects were asked to keep the arm outstretched during the experiments. The smooth curves of Fig. 3 were selected as templates to generate different stimulation patterns. During a trial, all vibro-actuators vibrate simultaneously. Single trial ran for an average of 80 milliseconds. During the first five trials, the template $(\mathrm{T})$ in Fig. 3 was played. Before playing each template, subjects were shown the printed template. Subjects were asked to draw a smooth curve representing what they perceive on an ipad sketching app (Draw free app (Apple Inc)) after each trial as shown in Fig. 2B. A drawing area on the ipad was clearly demarcated to match the size of the printed template as shown in Fig. 2B. Just after the drawing, the next stimulation was given. On average inter-stimulus interval was $82 \mathrm{~ms}$ on average with: $8.3 \mathrm{~ms}$ standard deviation. On average, experiment stimulation was limited to $80 \mathrm{~ms}$. After that period, subjects were free to start the drawing the estimated template.

\subsection{Data processing and statistical analysis}

The same available pencil in Draw free app (Apple Inc) was used for drawing throughout the experiments. Get Data Graph Digitizer version 2.6 was used to digitize the data ( 16 digits) on drawn lines. To obtain the regression coefficients, the respective template was generated by MATLAB 2014a with the exact length of the drawing curve for each trial. The regression coefficients were calculated between humans sketch data (raw data) and the respective templates. 

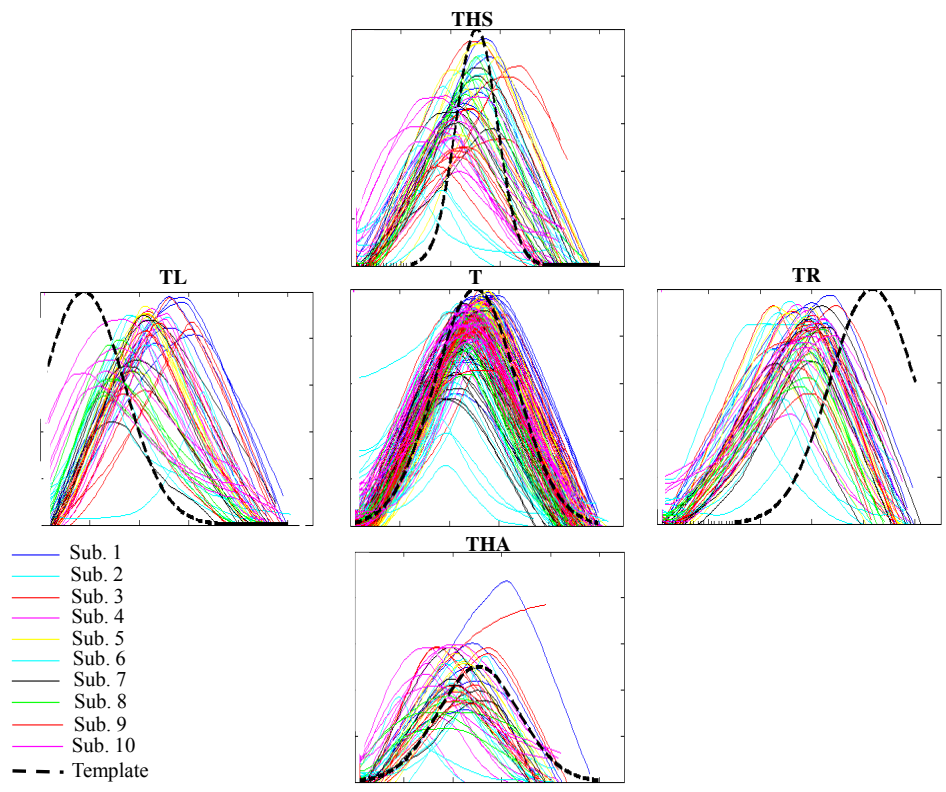

Fig. 4. The raw data for experiment 1: The sketched data for pattern T, TL, TR, THS, and THA for all trials. The all templates are shown by black dashed line.

\subsection{Experiment 1: To understand how humans generalize a Gaussian pattern in scaling and shifting}

Eight healthy naive subjects ( 6 - male, 2 - female) age between 24 to 39 participated in the experiment 1 . The experiment 1 was conducted to test how humans generalize a primitive template pattern $(\mathrm{T})$ with respect to scaling and shifting. The shifting was done by left shift (TL) and right shift (TR), not up or down and scaling was done by shrinking (THS), and half in magnitude (THA) as shown in Fig. 3.

In experiment 1 , subjects were asked to wear the vibro-actuator belt. Subjects were trained and shown only printed template $\mathrm{T}$. They were informed that the intensities of the vibro-actuators in the belt are directly proportional to the height of the template $\mathrm{T}$ during the stimulation. Subjects were told that they are supposed to draw a smooth curve with heights directly proportional to the intensities of the vibro-actuators after the stimulation. During the experiment, subjects were trained for only template $\mathrm{T}$. Therefore, at the beginning of the experiment, template $\mathrm{T}$ was played 5 times. Then subjects were informed that trained and untrained templates would be played pseudo randomly during the experiment. Therefore, after first five training trials, TL, TR, THS, and THA patterns were played randomly. However, there are training blocks in between 
A

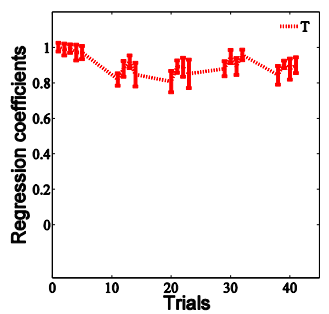

B

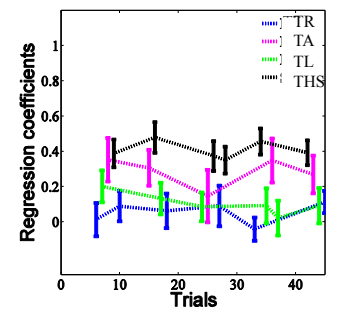

C

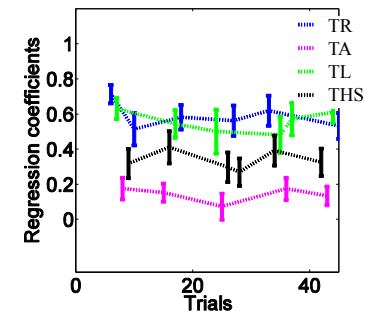

Fig. 5. Experiment 1: Average regression coefficients (In this experiment subjects were trained only for template $\mathrm{T}$ ): A) Regression coefficients for training template $\mathrm{T}$ over the trials, B) Testing session regression coefficients for templates TL, TR, THS, and THA when the data are regressed with its respective templates, and C) Regression coefficients for templates TL, TR, THS, and THA when the data are regressed with template $\mathrm{T}$.

other templates to train template $\mathrm{T}$ as shown in Table 2. During the training blocks, subjects were informed that the printed template was shown prior to the trial. For all training sessions (when $\mathrm{T}$ played) the visual cue was provided. At the end of each trial subjects were asked to draw what they felt during the trial. Pattern T repeated four times like in a block as shown in Table 2. Likewise four blocks of templates were played during the experiments after first five trials. The rest of four intensity patterns (TR, TL, THA, and THS) played six times each during the experiment randomly as shown in Table 2. Therefore, subjects participated in 45 trials during the experiments. For more clarity, the frequncies and the trial number and respective played patterns are shown in Table 1 and Table 2 respectively.

\subsection{Experiment 2: To understand how humans can recognize trained templates when they are presented in a random order}

The second experiment was conducted to test how subjects recognize all trained haptic feedback patterns when they are presented in a random order. Six healthy naive subjects ( 4 - male, 2 - female) aged 24 to 28 participated in the experiment 2. All instructions in experiment 1 were given to the subjects. However, not only the template $\mathrm{T}$, but also the templates TR, TL, and THA were shown to the subjects. Since the subjects were not able to distinguish the pattern THS from other patterns in experiment 1 , the pattern THS was dropped and only patterns TL, TR, THA, and T were considered for the experiment 2. During the experiment, first 20 trials were designed to train the subjects to learn the patterns T, TR, TL, and THA. Each training pattern was played 5 times. During those 20 training trials, subjects were shown the pattern to be played. Finally the subjects were asked to draw a smooth curve representing the vibro-actuator intensity pattern they felt on an ipad screen. During the testing session, the 

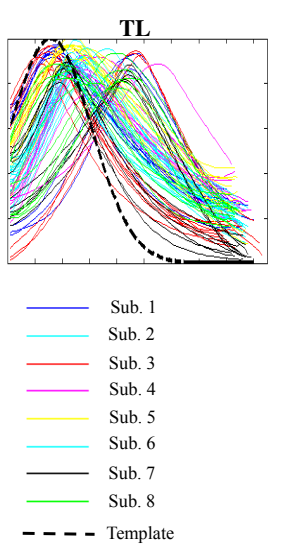

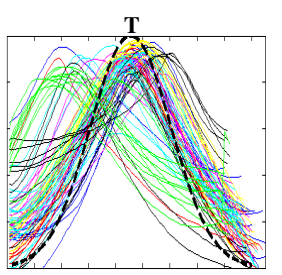

THA

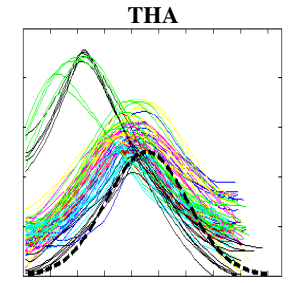

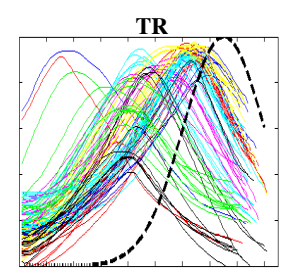

Fig. 6. The raw data for experiment 2(a): The sketched data for pattern T, TL, TR, and THA for all trials in experiment 2. The templates are shown by black dashed line. The template THS in experiment 1 was dropped in experiment 2.

four training templates were played in pseudo random order to achieve counter balancing. Each template was played 6 times making the total number of trials experienced by each subject to be 44 .

\section{Results}

\subsection{Experiment 1}

The sketched raw data for the pattern T, TL, TR, THS, and THA in experiment 1 are shown in Fig. 4 . The template patterns are shown by black dashed line. The sketched data in Fig. 4 were regressed by respective templates shown by black dashed line for each template. The average regression coefficients are shown in Fig. 5. The results in Fig. 5A show high correlationship between the data and template only when the template $\mathrm{T}$ was played. The regression coefficients of the first five trials in Fig. 5A confirms that this training block sets up a baseline.

Moving to the regression coefficients in templates TL, TR, THA, and THS regression coefficient values are relatively low when the sketched data are regressed with respective templates as shown in Fig. 5B. Moreover, it is noticed that some regression values less than 0 for templates TR and TL in Fig. 5B. The deviation can be noticed in humans' sketched data in Fig. 4 for templates TR and TL. However, the low and negative regression coefficients and higher variability values in Fig. $5 \mathrm{C}$ suggest that subjects were not able to shift the pattern (TL and TR) they are trained in. This might come from the fact that the memory of the pattern $\mathrm{T}$ interferes with subjects' perception as shown in raw data in Fig. 4. For more clarity, the data were regressed with template $\mathrm{T}$ 


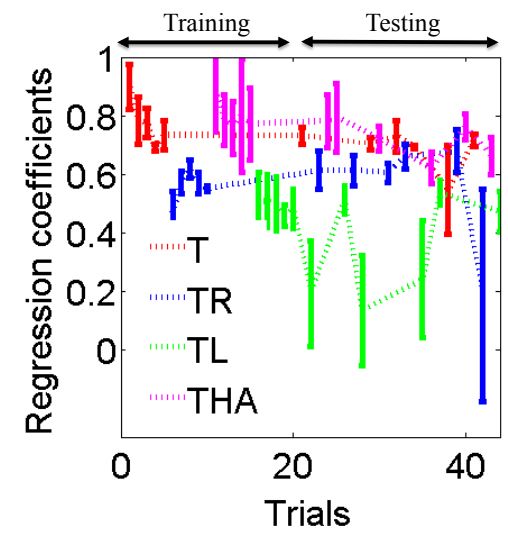

Fig. 7. A) Experiment 2: Average regression coefficients when data regressed with respective template in Fig. 3. Standard deviation of the regression coefficients are shown by error bars.

as shown in Fig. 5C (Note that in Fig. 5B the regression was done against the actual pattern that was played).

\subsection{Experiment 2}

The sketched raw data for the pattern T, TL, TR, and THA in experiment 2 are shown in Fig. 6 . The templates are shown by black dashed line. The sketched data in Fig. 6 were regressed against respective templates. The average regression coefficients are shown in Fig. 7 when subjects were trained and tested in a random order of patterns T, THA, TL, and TR in experiment 2. In general, in Fig. 7 all regression coefficients have improved with respect to Fig. 5B for all templates. The average regression coefficients of training session are higher for $\mathrm{T}$ and THA with respect to TL and TR as shown in Fig. 7. It implies that subjects have a better ability to recognize scaled template than shifted ones as noticed in experiment 1. Moreover, it can be seen in sketched data in Fig. 6 too. Interestingly, subjects can recognize these four templates when they are played in random order, provided they were trained earlier. Therefore, those results show that subjects can recognize trained primitive patterns when vibro-actuator array generates different stimulations.

\section{Discussion}

This paper presents experimental evidence of the capabilities and limitations of the human somatosensory system to distinguish and recognize a class of primitive haptic feedback pattern presented after prior training. Three consecutive 
experiments that provide insights into how humans recognize trained cutaneous feedback patterns as well as their shifts and scales. The results in this papper provide new insights into an important area of tactile input to inform shape recognition. The subjects' drawings of stimulus waveforms were captured and regression coefficients were used to understand humans' ability to recognize the given stimulations by different templates a via vibro-actuator array.

It would be interesting to test whether subjects could decode the messages from the wearable haptic sleeve when the user is mobile and active. It will inform to develop a vocabulary to be used in a haptic language for special information and to design training protocols.

A deeper understanding of humans' ability to generalize and recognize trained templates are important to convey some messages via vibro-actuator stimuli when humans have to work in noisy environments. Moreover, those trained templates could be considered as primitives of a haptic based language. Those primitives and subjects' responses would give an idea as to how they can be used to increase humans' perception when they are in noisy environments like factory, search and rescue.

Results of experiment 1 show that even though subjects were able to scale they find it difficult shift a trained template $T$. This results suggest that trained template $\mathrm{T}$ interfere for shifting. The raw data in experiment 1 shows higher deviation from the played template. Even for the template $\mathrm{T}$ in Fig. 5 has a higher deviation from the trained template $\mathrm{T}$. This variability could come from physiological factors like muscle tension and psychological factors like attention.

However, results in experiment 2 show that average regression coefficients have improved with respect to experiment 1 . These improvements show that subjects have an ability to recognize the same distributed haptic pattern (in this case T) even when it is shifted along the arm. Here TL is more precise and less strongly shifted to the center of the TR. It could come from that more sensitivity bias in shoulder than elbow (please note vibro-actuators labeled from 1 to 7 in Fig. 3 are from upper arm to lower arm.) as shown in raw data in Fig. 6. It would be useful to further investigate the distribution of sensitivity to vibro-tactile feedback along the human arm. Even though the same procedure was used for training for all experiments, the low regression coefficients in Fig. 5 and Fig. 7 suggest that even if recognition of the tactile patterns were good, performance would still be poor if drawing the visual representation was difficult.

The results explain as to how to use cutaneous feedback to the humans to convey some messages when humans working in noisy environments. It would help them to mentally construct the message by their training experiences as noticed in the results in experiments. The findings would give an insight about how special haptic memory is represented in the brain, how they could be linearly combined, and how humans can be trained for using multiple haptic patterns to decode complex messages. Those findings would be used to design guidelines/algorithm to convey messages to human in uncertain/noisy situations. 
Acknowledgments. The authors would like to thank UK Engineering and Physical Sciences Research Council (EPSRC) grant no. EP/I028765/1 and grant no. EP/NO3211X/1, the Guy's and St Thomas' Charity grant on developing clinician-scientific interfaces in robotic assisted surgery: translating technical innovation into improved clinical care (grant no. R090705), Higher Education Innovation Fund (HEIF), and Vattikuti foundation.

\section{References}

1. Hale, K. S., Stanney, K. M.: Deriving haptic design guidelines from human physiological, psychophysical, and neurological foundations, Computer Graphics and Applications, IEEE, vol. 24, no. 2, 33-39, (2004)

2. Gilson, R. D., Redden, E. S., Elliott, L. R., Remote tactile displays for future soldiers, tech. rep., DTIC Document (2007)

3. Jones, L. A., Lederman,S. J., Human hand function. Oxford University Press (2006)

4. Gilson, R. D., Redden, E. S., Elliott, L. R., Remote tactile displays for future soldiers, UNIVERSITY OF CENTRAL FLORIDA ORLANDO, (2007)

5. Tsukada, K., Yasumura, M., Activebelt: Belt-type wearable tactile display for directional navigation, Springer Berlin Heidelberg In UbiComp 2004: Ubiquitous Computing, 384-399, (2004)

6. Bliss, J. C., Katcher, M. H., Rogers, C. H., Shepard, R. P., Optical-to-tactile image conversion for the blind, IEEE Transactions on Man-Machine Systems, 11(1), 58-65, (1970)

7. Wall III, C., Weinberg, M. S., Schmidt, P. B., Krebs, D. E., Balance prosthesis based on micromechanical sensors using vibrotactile feedback of tilt, IEEE Transactions on Biomedical Engineering, 48(10), 1153-1161, (2001)

8. Priplata, A. A., Niemi, J. B., Harry, J. D., Lipsitz, L. A., Collins, J. J., Vibrating insoles and balance control in elderly people, The Lancet, 362(9390), 1123-1124, (2003)

9. Rupert, A. H., An instrumentation solution for reducing spatial disorientation mishaps, IEEE Engineering in Medicine and Biology Magazine, 19(2), 71-80, (2000)

10. Van Erp, J. B., Guidelines for the use of vibro-tactile displays in human computer interaction, IEEE In Proceedings of eurohaptics, pp. 18-22, (2002)

11. Stepanenko, Y., Sankar, T. S., Vibro-impact analysis of control systems with mechanical clearance and its application to robotic actuators, Journal of dynamic systems, measurement, and control, 108(1), 9-16, (1986)

12. Benali-Khoudja, M., Hafez, M., Alexandre, J. M., Khedda, A., Moreau, V., VITAL: a new low-cost vibro-tactile display system, IEEE International Conference onIn Robotics and Automation, 1, 721-726, (2004)

13. Zaitsev, V., Sas, P, Nonlinear response of a weakly damaged metal sample: a dissipative modulation mechanism of vibro-acoustic interaction, Journal of Vibration and Control, 6(6), 803-822, (2000)

14. Thoroughman, K.A., Shadmehr, R., Learning of action through adaptive combination of motor primitives, Nature, 407(6805), (2000) 